\title{
25 \\ Window based estimation of the intensity of a doubly stochastic Poisson process
}

\author{
J. Virtamo, S. Aalto and D. Down \\ VTT Information Technology \\ P.O.Box 1202, FIN-02044 VTT, Finland. Telephone: +35804565612. \\ Fax: +3580455 0115. email: jorma.virtamo@vtt.fi
}

\begin{abstract}
Tasks related to advanced management of telecommunications networks, such as optimal dynamic routing or dynamic allocation of bandwidth for virtual paths in an ATM network, call for a method to estimate the current call arrival intensities. Such an estimate must be based on the observed history of the call arrival instants. In this paper, we study window type estimators with the purpose of giving a clear idea of their accuracy. In particular, the simple straight case and exponential windows are considered and applied to an example where the correlation function of the intensity process is of the exponential type - a system for which the exponential window, in fact, is the optimal window. It turns out that the accuracy is controlled by a single dimensionless traffic parameter. The achievable accuracy is little affected by the shape of the window function. It also turns out that a Bayesian approach, applied in an earlier study, gives only a slight improvement over the window based estimator. More important instead is the proper choice of the size of the window. In order to find the optimal size, one has to know some basic characteristics of the intensity process. We briefly address the problem how these can be inferred from the long term statistics of the counting process.
\end{abstract}

\section{Keywords}

estimation, call arrival intensity, window, kernel, doubly stochastic Poisson process

\section{INTRODUCTION}

Many of the advanced management schemes of telecommunications networks assume that the current call arrival intensity $\lambda$ of any given traffic stream is known. Such information is needed e.g. for optimal dynamic routing in telephone networks (Krishnan, 1990, Mitra et al., 1993) or dynamic forward-looking allocation of bandwidth for virtual paths in an ATM network (Bruni et al., 1994). In practice, of course, call arrival intensities are not given, and as a separate problem one must estimate their values on the basis of the available information. Typically one can assume that the arrival process constitutes a 
doubly stochastic Poisson process, i.e. a Poisson process where the intensity $\lambda_{t}$ is also a stochastic process. What is observed is the point process of call arrivals (counting process $N_{t}$ ) and the task is then to estimate $\lambda_{t}$ based on these observations. Such a real-time traffic estimation problem is essentially a filtering problem (Warfield et al., 1994).

In (Aalto and Virtamo, 1995) we developed such an algorithm for the case where the nature of the process $\lambda_{t}$ was fully known in advance. In particular, a Bayesian estimator was developed with the assumption that $\lambda_{t}$ is an Ornstein-Uhlenbeck process. This estimator exploits to a maximum degree all the available information and in that sense is an optimal estimator. Though the algorithm presented was not complex, one might desire an even simpler algorithm. Moreover, the particular process model does not have general validity, and it would be desirable to have a scheme which does not depend on specific assumptions on the underlying process.

The most straightforward estimators are based on a sliding window, i.e. on a count of past arrivals within a window divided by its length, possibly with different weights in different parts of the window. In this paper, our aim is to give a clear idea on the accuracy of window estimators. The problem belongs to the area of linear filtering theory and has been subject to earlier analysis (Snyder, 1975).

In section 2 we rederive a formula for the mean squared error of the estimator. This turns out to depend only on the correlation function of the process $\lambda_{t}$ (in addition to the mean and variance of the stationary distribution of $\lambda_{t}$ ) but not on other dynamic characteristics of the process. Two different window types are considered in section 3: a straight case window and an exponential window. The latter is the most attractive one from the algorithmic point of view. Besides the standard window estimator, we consider an improved estimator which exploits the prior knowledge of the mean of $\lambda_{t}$, using a linear combination of this prior value and the window "measurement" as the actual estimator. The optimal mixture of these two components is derived. This improved estimator is indeed found to increase the accuracy, especially in those cases where the accuracy of a pure window estimator is poor. In all the cases the achievable accuracy is controlled by a single traffic parameter.

In section 4 we consider the problem of determining the optimal window function. This satisfies a known integral equation (Snyder, 1975, Van Trees, 1971). We give a general solution to this equation in terms of Fourier transforms. For an exponential correlation function the optimal window function itself turns out to be of exponential type. In section 5 , the problem of inferring the characteristics of the underlying intensity process from the observed Poisson counting process is briefly addressed. The same dimensionless parameter which governs the accuracy of the estimator also determines the accuracy of the long term measurements. Finally, numerical results are presented and discussed in section 6 .

\section{ANALYSIS}

The intensity process $\lambda_{t}$ is assumed to be stationary with mean $\Lambda$ and variance $\Sigma^{2}$. Its covariance function is denoted by $\Sigma^{2}(t)$ and can be expressed in terms of the correlation function $\rho(t)$,

$\operatorname{Cov}\left[\lambda_{t}, \lambda_{u}\right]=\Sigma^{2}(t-u)=\Sigma^{2} \rho(t-u)$. 
The covariance is symmetric in its variables. Therefore, the correlation function is a symmetric function, i.e. $\rho(t)=\rho(-t)$.

Let $N_{t}$ be the counting process of the arrivals. A window based estimator $X$ for the arrival intensity $\lambda_{0}$ at time 0 is

$X=\int_{0}^{\infty} W(t) \mathrm{d} N_{t}=\sum_{i} W\left(t_{i}\right)$

where $W(t)$ is the window function such that $\int_{0}^{\infty} W(t) \mathrm{d} t=1$ and the $t_{i}$ are the arrival instants. (For notational simplicity we use here reversed time). $X$ itself is, of course, a stochastic variable. Its mean is

$\mathrm{E}[X]=\int_{0}^{\infty} W(t) \mathrm{E}\left[\mathrm{d} N_{t}\right]=\int_{0}^{\infty} W(t) \Lambda \mathrm{d} t=\Lambda$.

In choosing the window function one has to balance between two trends: if the function is concentrated around time 0 the estimator is based on a few recent arrivals and can be very noisy; on the other hand broadening the window too much means that the estimator is based on outdated information.

The estimator can be improved by taking it to be of more general linear form

$Y=a X+a^{\prime}$

Let us consider the error $\Delta$ of the estimator

$\Delta=Y-\lambda_{0}=a X+\left(a^{\prime}-\lambda_{0}\right)$.

We wish to choose the window function $W(t)$ and parameters $a$ and $a^{\prime}$ such that the mean squared error

$\mathrm{E}\left[\Delta^{2}\right]=\mathrm{D}^{2}[\Delta]+\mathrm{E}[\Delta]^{2}$

is minimized. Since the variance of $\Delta$ in (4) is not affected by the constant $a^{\prime}$, the optimal choice of $a^{\prime}$ makes the estimator unbiased, i.e.

$\mathrm{E}[\Delta]=(a-1) \Lambda+a^{\prime}=0$

from which

$a^{\prime}=(1-a) \Lambda$.

With this value we can write

$Y=a(X-\Lambda)+\Lambda, \quad \Delta=a X-\lambda_{0}+(1-a) \Lambda$

i.e. the estimator is $\Lambda$ plus a correction term where the deviation of the "measured" value $X$ from $\Lambda$ is multiplied by a constant $a$. The optimal choice of $a$ obviously depends on the confidence of the measurement. With full confidence we have $a=1$ and $Y=X$. 
Now, because $\mathrm{E}[\Delta]=0$, we have using (5)

$\mathrm{E}\left[\Delta^{2}\right]=\mathrm{D}^{2}[\Delta]=\mathrm{D}^{2}\left[a X-\lambda_{0}\right]=a^{2} \mathrm{D}^{2}[X]-2 a \operatorname{Cov}\left[X, \lambda_{0}\right]+\mathrm{D}^{2}\left[\lambda_{0}\right]$.

Let us calculate each of the terms. First,

$\mathrm{D}^{2}[X]=\int_{0}^{\infty} W^{2}(t) \mathrm{D}^{2}\left[\mathrm{~d} N_{t}\right]+\int_{0}^{\infty} \int_{0}^{\infty} W(t) W(u) \operatorname{Cov}\left[\mathrm{d} N_{t}, \mathrm{~d} N_{u}\right]$

Here, because $\mathrm{d} N_{t}^{2}=\mathrm{d} N_{t}$,

$\mathrm{D}^{2}\left[\mathrm{~d} N_{t}\right]=\mathrm{E}\left[\mathrm{d} N_{t}\right]-\mathrm{E}^{2}\left[\mathrm{~d} N_{t}\right]=\Lambda \mathrm{d} t-(\Lambda \mathrm{d} t)^{2} \rightarrow \Lambda \mathrm{d} t$,

and

$\operatorname{Cov}\left[\mathrm{d} N_{t}, \mathrm{~d} N_{u}\right]=\operatorname{Cov}\left[\lambda_{t}, \lambda_{u}\right] \mathrm{d} t \mathrm{~d} u$.

Thus,

$\mathrm{D}^{2}[X]=\Lambda \int_{0}^{\infty} W^{2}(t) \mathrm{d} t+\int_{0}^{\infty} \int_{0}^{\infty} W(t) W(u) \operatorname{Cov}\left[\lambda_{t}, \lambda_{u}\right] \mathrm{d} t \mathrm{~d} u$.

Second,

$\operatorname{Cov}\left[X, \lambda_{0}\right]=\int_{0}^{\infty} W(t) \operatorname{Cov}\left[d N_{t}, \lambda_{0}\right]=\int_{0}^{\infty} W(t) \operatorname{Cov}\left[\lambda_{0}, \lambda_{t}\right] \mathrm{d} t$

Third, $\mathrm{D}^{2}\left[\lambda_{0}\right]=\Sigma^{2}$. By collecting the results together and using the correlation function (1), we have

$\frac{\mathrm{E}\left[\Delta^{2}\right]}{\Sigma^{2}}=1+a^{2}\left(\beta I_{1}+I_{2}\right)-2 a I_{3}$

where $\beta=\Lambda / \Sigma^{2}$ and $I_{1}, I_{2}$ and $I_{3}$ stand for the integrals

$I_{1}=\int_{0}^{\infty} W^{2}(t) \mathrm{d} t, \quad I_{2}=\int_{0}^{\infty} V(t) \rho(t) \mathrm{d} t, \quad I_{3}=\int_{0}^{\infty} W(t) \rho(t) \mathrm{d} t$,

and

$V(t)=2 \int_{0}^{\infty} W(u) W(u+t) \mathrm{d} t$.

The standard window estimator $X$ is obtained by setting $a=1$

$\frac{\mathrm{E}\left[\Delta_{X}^{2}\right]}{\Sigma^{2}}=1+\beta I_{1}+I_{2}-2 I_{3}$.

A better estimator is obtained by minimizing (6) with respect to $a$,

$\frac{\mathrm{E}\left[\Delta_{Y}^{2}\right]}{\Sigma^{2}}=1-\frac{I_{3}^{2}}{\beta I_{1}+I_{2}}, \quad$ with $\quad a^{*}=\frac{I_{3}}{\beta I_{1}+I_{2}}$. 
Note that the results depend on $\Lambda$ and $\Sigma$ only through the single parameter $\beta=\Lambda / \Sigma^{2}$.

\section{EXAMPLES}

In this section we consider the problems where the form of the window function $W(t)$ is given but it contains one free parameter, $T$, which characterizes the temporal extent of the window (loosely speaking the window size) and a further minimization is performed with respect to this parameter. In particular, the window function is assumed to be either a straight case window or an exponential window.

\subsection{Straight case window}

In this case the window function is

$W(t)=\frac{1}{T} U(t / T)$

where $U(x)$ is the unit step function, i.e. $U(x)=1$ for $0 \leq x<1$ and 0 otherwise. The window estimator (2) then is

$X=\frac{1}{T} \sum_{i} 1_{t_{i}<T}$

i.e. the count of arrivals in the window divided by the length of the window. This is algorithmically a simple formula. However, one has to keep a list of the arrival times, and an entry in the list cannot be deleted until time $T$ has elapsed since the corresponding arrival time. From the standard window estimator $X$ one then obtains the optimal estimator using (5).

With this window function, the integrals (7) become

$I_{1}=\frac{1}{T}, \quad I_{2}=\frac{2}{T} \int_{0}^{T}(1-t / T) \rho(t) \mathrm{d} t, \quad I_{3}=\frac{1}{T} \int_{0}^{T} \rho(t) \mathrm{d} t$.

This is as far as one can get in the general form. To proceed, one has to specify the correlation function $\rho(t)$. As an example we consider the following correlation function,

$\rho(t)=e^{-|t / \tau|}$

where $\tau$ is the correlation time. Such an exponential form is valid for e.g. the OrnsteinUhlenbeck process considered in (Aalto and Virtamo, 1995). By denoting $x=T / \tau$ we get with this correlation function

$I_{2}=\frac{2}{x^{2}}\left(e^{-x}-1+x\right), \quad I_{3}=\frac{1}{x}\left(1-e^{-x}\right)$. 
Substitution into (8) and (9) gives the mean squared error of the standard and the optimal estimators $X$ and $Y$,

$\frac{\mathrm{E}\left[\Delta_{X}^{2}\right]}{\Sigma^{2}}=1-2 \cdot \frac{1-b x-(1+x) e^{-x}}{x^{2}}, \quad \frac{\mathrm{E}\left[\Delta_{Y}^{2}\right]}{\Sigma^{2}}=1-\frac{1}{2} \cdot \frac{\left(1-e^{-x}\right)^{2}}{b x+e^{-x}-1+x}$,

with the optimum obtained at

$a^{*}=\frac{x}{2} \cdot \frac{1-e^{-x}}{b x+e^{-x}-1+x}$.

In these formulae $b$ is the dimensionless parameter

$b=\frac{\Lambda}{2 \Sigma^{2} \tau}=\frac{1}{2} \cdot \frac{1}{(\Sigma / \Lambda)^{2}} \cdot \frac{1}{\Lambda \tau}$.

The expressions in (11) are still to be minimized with respect to the parameter $x$, i.e. the window size. This cannot be done in analytic form: zero points of the derivatives have to be found by numerical methods. Nevertheless, the result of the minimization is solely a function of $b$, i.e. this parameter alone determines the achievable accuracy of the estimator.

\subsection{Exponential window}

Now the window function is

$W(t)=\frac{1}{T} e^{-t / T}$.

This is particularly attractive from the point of view of algorithmic simplicity. From eqs. (2) and (13) we have

$X=\frac{1}{T} \sum_{i} e^{-t_{i} / T}$

Thus, by advancing the time by an interval $\Delta t$ without arrivals, $X$ is multiplied by a factor $\exp (-\Delta t / T)$ (i.e. by a constant for fixed intervals), and a new arrival at time 0 increments its value by $1 / T$. There is no need to record the individual arrival times. As before, the optimal estimator is calculated from (5) with $X$ given by the above algorithm.

With this window function the integrals are

$I_{1}=\frac{1}{2 T}, \quad I_{2}=I_{3}=\frac{1}{T} \int_{0}^{\infty} e^{-t / T} \rho(t) \mathrm{d} t$,

and in the specific case of the exponential correlation function (10) we get (with $x=T / \tau$ )

$I_{2}=I_{3}=\frac{1}{1+x}$. 
The mean squared errors (8) and (9) of the standard and the optimal estimators are

$\frac{\mathrm{E}\left[\Delta_{X}^{2}\right]}{\Sigma^{2}}=1+\frac{b}{x}-\frac{1}{1+x}, \quad \frac{\mathrm{E}\left[\Delta_{Y}^{2}\right]}{\Sigma^{2}}=1-\frac{1}{1+x} \cdot \frac{x}{x+b(1+x)}$,

where the parameter $b$ is given by (12) and the optimum is obtained at $a^{*}=x /(x+b(1+$ $x)$ ).

As expressions in (14) are rational functions of $x$ the minimization with respect to this parameter can be done analytically giving the optimal window size for the standard estimator $X$ and for the optimal estimator $Y$

$x_{X}^{*}=\frac{\sqrt{b}}{1-\sqrt{b}}, \quad x_{Y}^{*}=\sqrt{\frac{b}{1+b}}$.

Finally, substituting these optimal window sizes into (14) gives the mean squared errors,

$\frac{\mathrm{E}\left[\Delta_{X}^{2}\right]}{\Sigma^{2}}=1-(1-\sqrt{b})^{2}, \quad \frac{\mathrm{E}\left[\Delta_{Y}^{2}\right]}{\Sigma^{2}}=1-\frac{1}{(\sqrt{b}+\sqrt{1+b})^{2}}$,

with the optimal $a$ being

$a^{*}=1-\sqrt{\frac{b}{1+b}}$

In the next section we will show that for the exponential correlation function the exponential window estimator $Y$ with the optimal parameter values $x_{Y}^{*}$ of (15) and $a^{*}$ of (17) indeed is the optimal estimator also with respect to the form of the wndow function.

\section{OPTIMAL WINDOW}

Thus far we have considered window estimators with a given form of the window function. In order to find the truly optimal window estimator the form of the window should also be subject to optimization. For the present purposes it is most convenient to absorb even the coefficient $a$ in (3) into the definiton of the window function, i.e. here the window function $W(t)$ is not normed but both its shape and norm are simultaneously optimized. The optimal $a$ is then regained just as the norm of the optimal window function, and the best value for the constant $a^{\prime}$ in (3) is as before $(1-a) \Lambda$. It can be shown (Snyder, 1975) that the optimal window function $W(t)$ satisfies the following integral equation

$$
\beta W(t)+\int_{0}^{\infty} W(u) \rho(t-u) \mathrm{d} u=\rho(t), \quad t \geq 0
$$

This integral equation arises naturally in the study of linear filters for the detection of signals in the presence of additive noise, see for example (Van Trees, 1971). In fact, it is described in (Snyder, 1975) how determining the optimal window' may be recast as such a detection problem. There it is also discussed how such problerns may be treated 
with various tools from linear filtering theory, including a Kalman-Bucy approach. Here we will simply indicate how the solution may be obtained directly by transform methods. Let us define the Fourier transform $f^{*}(k)$ of a function of time $f(t)$

$f^{*}(k)=\int_{-\infty}^{\infty} e^{-i k t} f(t) \mathrm{d} t$

Define

$f_{+}(t)=1_{t \geq 0} f(t), \quad f_{-}(t)=1_{t<0} f(t)$,

and denote

$S(t)=\int_{0}^{\infty} W(u) \rho(t-u) \mathrm{d} u=\int_{-\infty}^{\infty} W_{+}(u) \rho(t-u) \mathrm{d} u=W_{+} \otimes \rho$

with $\otimes$ representing the convolution operation. Equation (18) may now be rewritten as

$\beta W_{+}(t)+S_{+}(t)=\rho_{+}(t), \quad \forall t$.

Adding $S_{-}(t)$ to both sides of (19) and taking Fourier transforms yields

$W_{+}^{*}(k)=\frac{\rho_{+}^{*}(k)+S_{-}^{*}(k)}{\beta+\rho^{*}(k)}$.

It can be seen that the only singularities of $W_{+}^{*}(k)$ in the upper half plane are the roots of $\beta+\rho^{*}(k)=0$. The fact that $\rho^{*}(k)=\rho_{+}^{*}(k)+\rho_{+}^{*}(-k)$ implies that these roots appear in conjugate pairs. Let us denote them by $\pm i \kappa_{j}$ with $\kappa_{j}>0$.

We can now formally write the inverse transform as

$W(t)=i \sum_{j} \operatorname{res}\left\{\frac{\rho_{+}^{*}(k)+S_{-}^{*}(k)}{\beta+\rho^{*}(k)}\right\}_{k=i \kappa_{j}} e^{-\kappa_{j} t}$.

The function $S_{-}^{*}(k)$ may be determined from the condition that expression (20) as a whole represents the Fourier transform of a "+ function" and thus is analytic in the lower half plane. The function $S_{-}^{*}(k)$ must be such that the numerator has roots at $k=-i \kappa_{j}$ in order to cancel the roots of the denominator in the lower half plane.

Let us again consider the example of the exponential correlation function $\rho(t)=e^{-|t / \tau|}$. We use $\tau$ as the unit of time and, as before, denote $b=\beta / 2 \tau$. Then we have

$\rho_{+}^{*}(k)=\frac{1}{1+i k}, \quad \rho^{*}(k)=\frac{2}{1+k^{2}}, \quad \kappa=\sqrt{1+\frac{1}{b}}$

where we have dropped the subscript as there is only one pair of roots $\pm i \kappa$. We can easily calculate that

$S_{-}^{*}(k)=\frac{C}{1-i k}, \quad C=\int_{0}^{\infty} \mathrm{d} t W_{+}(t) e^{-t}$. 
Then, from (21),

$W(t)=i \cdot \operatorname{res}\left\{\frac{1-i k+C(1+i k)}{2 b(k-i \kappa)(k+i \kappa)}\right\}_{k=i \kappa} e^{-\kappa t}$.

The constant $C$ can now be determined by requiring that the numerator has a root at $-i \kappa$ from which it follows that $C=(\kappa-1) /(\kappa+1)$, and $W(t)=a \kappa e^{-\kappa t}$, where $W(t)$ is written in the form of a constant times a normed function with

$a=1-\frac{1}{\kappa}=1-\sqrt{\frac{b}{1+b}}$.

Thus we have regained the optimal solution $Y$ of section 3.2 with an exponential window: an exponential window is the optimal window for the case of an exponential correlation function. Finally, we can easily check that the constant $C$ is defined consistently, i.e. the substitution of $W(t)$ into the defining integral of $C$ yields $(\kappa-1) /(\kappa+1)$.

\section{INFERRING THE CHARACTERISTICS OF THE INTENSITY PROCESS FROM THE COUNTING STATISTICS}

All the results presented above were based on the assumption that the basic characteristics of the intensity process were known, viz. its mean $\Lambda$, variance $\Sigma^{2}$ and the covariance function $\Sigma^{2}(t)$. Information on these can be obtained only indirectly via the long term statistics of the counting process. An estimate for the mean $\Lambda$ is of course provided by the mean count per time interval $\lim _{t \rightarrow \infty} N_{t} / t$. The covariance function can also, in principle, be obtained straightforwardly by determining the counting statistics $\mathrm{E}\left[\mathrm{d} N_{u} \mathrm{~d} N_{v}\right]$ of two non-overlapping intervals $\mathrm{d} u$ and $\mathrm{d} v$, with

$\mathrm{E}\left[\mathrm{d} N_{u} \mathrm{~d} N_{v}\right] \approx\left(\operatorname{Cov}\left[\lambda_{u}, \lambda_{v}\right]+\Lambda^{2}\right) \mathrm{d} u \mathrm{~d} v$.

The problem with this is that in order to make the points of time $u$ and $v$ precise, $\mathrm{d} u$ and $\mathrm{d} v$ should be chosen small. This implies a poor statistics or very long measurement periods to get a reliable expectation in the above formula.

A better approach is to determine experimentally $\mathrm{D}^{2}\left[N_{t}\right]$ as a function of $t$ and derive the covariance function from this function. To this end let us denote

$n_{t}=\int_{0}^{t} \lambda_{t} \mathrm{~d} t$

Given the value of $n_{t}$ the variable $N_{t}$ is Poisson distributed with mean $n_{t}$. Therefore we have

$\mathrm{E}\left[N_{t}\right]=\mathrm{E}\left[\mathrm{E}\left[N_{t} \mid n_{t}\right]\right]=\mathrm{E}\left[n_{t}\right]=\Lambda t, \quad \mathrm{D}^{2}\left[N_{t}\right]=\mathrm{E}\left[n_{t}\right]+\mathrm{D}^{2}\left[n_{t}\right]$.

From the definition of $n_{t}$ we can easily deduce that

$\mathrm{D}^{2}\left[n_{t}\right]=\int_{0}^{t} \mathrm{~d} u \int_{0}^{t} \mathrm{~d} v \Sigma^{2}(u-v)=2 \int_{0}^{t} \mathrm{~d} u \int_{0}^{u} \mathrm{~d} v \Sigma^{2}(v)$ 


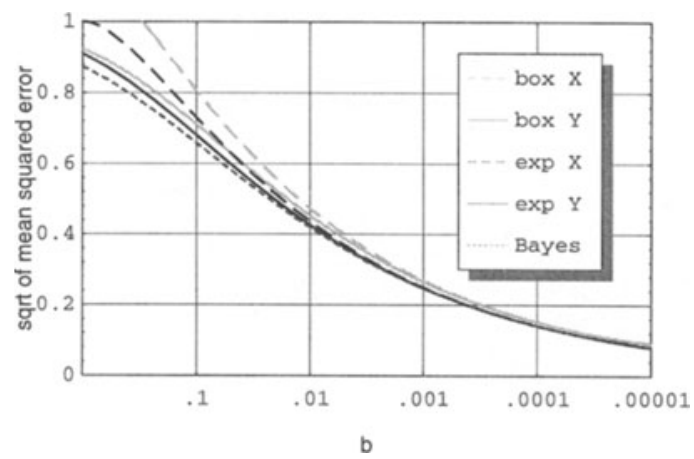

Figure 1 Accuracy of the estimators.

and thus

$\mathrm{D}^{2}\left[N_{t}\right]=\Lambda t+2 \int_{0}^{t} \mathrm{~d} u \int_{0}^{u} \mathrm{~d} v \Sigma^{2}(v)$.

Differentiating, we then have

$\frac{d}{d t} \mathrm{D}^{2}\left[N_{t}\right]=\Lambda+2 \int_{0}^{t} \Sigma^{2}(u) \mathrm{d} u, \quad \frac{d^{2}}{d t^{2}} \mathrm{D}^{2}\left[N_{t}\right]=2 \Sigma^{2}(t)$.

The latter relation can be used for determining the covariance function. However, a numerical evaluation of the second derivative is involved which is susceptible to noise. This is a reflection of the same problem we alluded to in discussing the direct measurement of the covariance function. A more stable procedure would entail choosing a parameterized functional form for $\Sigma^{2}(t)$ and determining the parameters by a global fit to (23).

\section{NUMERICAL RESULTS AND DISCUSSION}

Numerical results of the accuracy $\sqrt{\mathrm{E}\left[\Delta^{2}\right]} / \Sigma$ of different estimators as a function of $b$ are shown in Figure 1. These were obtained from (11) in the case of a straight case window, with the additional numerical optimization with respect to the window size $x$, and from (16) in the case of an exponential window. For comparison, the accuracy of a Bayesian estimator (for which an approximation was derived in (Aalto and Virtamo, 1995)) is also shown. The latter is based on full information about the process model of $\lambda_{t}$, which here is assumed to obey the Ornstein-Uhlenbeck process (exponential correlation function). The accuracy of the Bayesian estimator is not solely a function of $b$ but depends separately on $\Lambda \tau$ and $\Sigma / \Lambda$. Here we have plotted the curve for the case where $\Sigma / \Lambda$ is kept fixed to 0.3 and $b=1 /\left(2(\Sigma / \Lambda)^{2}(\Lambda \tau)\right)$ is controlled by the value of $\Lambda \tau$.

First we note that the Bayesian estimator is the best one, as it should by construction. Second, the estimators with an exponential window function are more accurate than their straight case window counterparts. For the case of the estimator $Y$ this is obvious because 


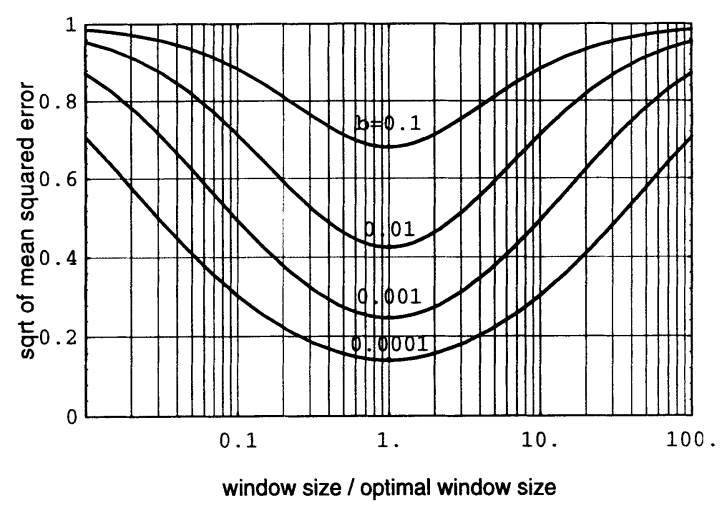

Figure 2 Sensitivity of the accuracy of the estimator $Y$ with respect to the window size.

we have shown that the exponential window is the optimal window. For very small $b$, the accuracies of the estimators differ only depending on the window function ( $X$ and $Y$ are identical). An exponential window function gives an estimator which is asymptotically as good as the Bayesian scheme. For values of $b$ closer to 1 , the more important factor is whether one uses the simple window estimator or the improved one with a mixture of the mean value $\Lambda$ and the window estimator.

In general, the differences between different estimators are relatively small. The main differences appear in the range of large values of $b$ where the accuracies of all the estimators are poor. When $b$ is small the estimators are more accurate and essentially the same for all the schemes. However, it should be noted that these results were obtained with the optimal window size. With a wrong window size the accuracy can deteriorate. This is shown in Figure 2 where the accuracy of the estimator $Y$ for an exponential window, given by (14), is plotted against the ratio of the window size to the optimal window size for different values of $b$. An order of magnitude error in the window size typically doubles the standard deviation of the estimator.

A somewhat surprising finding is that the achievable accuracy for the estimator does not change dramatically over a wide range of values of $b$, i.e. even though $b$ changes by five orders of magnitude the standard deviation of the estimator changes only by a factor of ten. By local observations of the doubly stochastic Poisson process it is hard to increase the accuracy over the estimate which is provided by the mean intensity determined from long term statistics.

Returning to the motivation of our work by the needs of dynamic resource management schemes, it should be noted that an estimate of the traffic intensity contains inherent inaccuracies. At best, the accuracy is determined by the mean squared error such as given by (16). However, errors in the long term measurements of the basic parameters can lead to the choice of a non-optimal window size, and consequently the estimation error may be larger. For the sake of the robustness of a management scheme relying on the knowledge of the call arrival intensities, the applied algorithms should explicitly take into account the uncertainty involved in the estimator. 


\section{REFERENCES}

Aalto, S. and Virtamo, J.T. (1995) Real-time estimation of call arrival intensities, in the proceedings of the seminar ATM hot topics on traffic and performance: from RACE to ACTS, Milano.

Bruni, C., D'Andrea, P., Mocci, U. and Scoglio, C. (1994) Optimal capacity assignment of virtual paths in ATM networks, Globecom'94, San Francisco, pp. 207-11.

Krishnan, K.R. (1990) Markov decision algorithms for dynamic routing. IEEE Communications Magazine, 28, 66-9.

Mitra, D., Gibbens, R.J. and Huang, B.D. (1993) State-dependent routing on symmetric loss networks with trunk reservations - I, IEEE Transactions on Communications, 41, 400-11.

Snyder, D.L. (1975) Random Point Processes. John Wiley \& Sons, New York.

Van Trees, H.L. (1971) Detection, Estimation, and Modulation Theory: Part III. John Wiley \& Sons, New York.

Warfield, R., Chan, S., Konheim, A. and Guillaume, A. (1994) Real-time traffic estimation in ATM networks, The Fundamental Role of Teletraffic in the Evolution of Telecommunications Networks (ed. J. Laboutelle and J.W. Roberts), the 14th International Teletraffic Congress - ITC14. Elsevier, Amsterdam, pp. 907-16. 